\title{
A Pan-Cancer Analysis of the Role of Selenoprotein $P$ mRNA in Tumorigenesis
}

\author{
Yanni Yang ${ }^{1-3, *}$ \\ Daning $\mathrm{Li}^{1, *}$ \\ Wentao Wu (iD) \\ Dingxing Huang ${ }^{\prime}$ \\ Haishi Zheng ${ }^{3}$ \\ Yirixiati Aihaiti ${ }^{3}$ \\ 'School of Public Health, Xi'an Jiaotong \\ University Health Science Center, Xi'an, \\ Shaanxi, People's Republic of China; \\ ${ }^{2}$ Shaanxi University of Traditional \\ Chinese Medicine, Xianyang, Shaanxi, \\ People's Republic of China; ${ }^{3}$ Department \\ of Joint Surgery, Xi'an Jiaotong University \\ Affiliated HongHui Hospital, Xi'an, \\ Shaanxi, People's Republic of China \\ *These authors contributed equally to \\ this work
}

Correspondence: Wentao Wu School of Public Health, Xi'an Jiaotong University Health Science Center, 76 Yanta West Road, Xi'an, Shaanxi, 71006I,

People's Republic of China

Tel +86 I56808337I6

Email15680833716@I63.com
Background: Selenium (Se) exhibits its anti-carcinogenic properties by regulating the redox system. However, the relationship between selenoprotein $\mathrm{P}$ (SeP), mRNA (SELENOP mRNA) and tumorigenesis remains unclear. Plasma SeP transports Se to various target tissues and has antioxidant characteristics. The present study aimed to explore the multifaceted pan-cancer properties of SELENOP in terms of its tissue-specific expression, prognostic value, immune function, and signaling pathway enrichment.

Patients and Methods: The expression profile of SELENOP was determined in 33 tumor types and survival, pathway enrichment, and correlation analyses were conducted based on TCGA database. The relationship between SELENOP expression and immune infiltration and macrophage subtype gene markers was investigated using the TIMER and GEPIA.

Results: SELENOP gene expression was decreased in many cancer tissues, but was upregulated in brain lower grade glioma (LGG). Furthermore, SELENOP expression was associated with a better prognosis in most cancers, but a poorer prognosis in LGG and uterine corpus endometrioid carcinoma (UCEC). Our results showed that SELENOP was correlated with infiltration level of six immune cell types, where SELENOP also showed a strong correlation with macrophages in some cancer types. However, we failed to determine macrophage polarization in 33 tumor types. SELENOP negatively regulated vascular endothelial cell proliferation in LGG and UCEC and epidermal cell differentiation in six tumor types. In contrast, upregulation was related to immune function, including $\mathrm{T}$ cell activation, B cell-mediated immunity, adaptive immune response and immune response regulation cell surface receptor signaling pathways in another six tumor types.

Conclusion: These findings highlighted the tissue-specific expression, prognostic value and immune characteristics of SELENOP in pan-cancer, and provided insights for illustrating the role of SELENOP in tumorigenesis.

Keywords: selenoprotein $\mathrm{P}$, pan-cancer, immune infiltration, prognosis, tissue-specific expression

\section{Introduction}

Selenium (Se) is a trace element that plays a crucial role in human health. Its biological function is mainly attributed to its presence in selenoproteins. ${ }^{1} \mathrm{Se}$ incorporates into the protein via the amino acid selenocysteine $(\mathrm{Sec}){ }^{2}$ Se metabolic system plays a key role in this process. Dietary Se is absorbed and the liver synthesizes and exports selenoprotein $\mathrm{P}$ (SeP). Then, SeP (encoded by SELENOP) circulates in the blood to transport Se to other tissues and organs. ${ }^{3}$ The transported Se is converted into intracellular selenophosphate, which is used to transform tRNA ${ }^{[\mathrm{ser}] \mathrm{sec}}$-bound serine into Sec. ${ }^{3,4} \mathrm{Sec}_{\mathrm{tRNA}}{ }^{[\mathrm{ser}] \mathrm{sec}}$ is incorporated 
into polypeptide chains, which finally completes the selenoprotein synthesis. ${ }^{4}$ Due to their special physiological domains, selenoproteins exhibit antioxidant activity and regulate Se transport, immune response and thyroid hormone metabolism. ${ }^{5}$ Oxidative damage has been demonstrated to play a carcinogenic role. Thus, Se demonstrates its anti-carcinogenic properties by regulating the redox system. $^{6,7}$

Several epidemiological studies have also revealed the benefits of Se in cancer. ${ }^{8-10}$ However, an excessive supply of Se may have adverse effects on brain diseases, endocrine system, and cancer. ${ }^{11}$ It is worth noting that Se may play several roles in the initiation and development of cancer. Our previous study showed a dual relationship between certain types of cancer and glutathione peroxidases (GPXs) and thioredoxin reductases (TXNRDs) in selenoproteins. $^{12}$

Although Se has a broad anti-carcinogenic effect, specific selenoproteins' contributions to the development of cancer remain unclear. With the rapid development of genomic technology, 25 genes encoding selenoproteins have been found in the human genome. ${ }^{1}$ Among them, SeP is a unique plasma selenoprotein, which contains more than one $\mathrm{Sec}$, while human SELENOP sequence has as many as ten Sec residues. ${ }^{13}$ Therefore, SeP is essential for Se transport to the targeted tissues. ${ }^{14} \mathrm{SeP}$ is mainly synthesized in the liver, but it is also expressed in other organs, particularly the brain, kidney and testis. ${ }^{15,16}$ In addition to its transport function, SELENOP also has antioxidant properties in the development of diseases, such as colitis-associated and non-small cell lung cancers. ${ }^{14,17}$ SELENOP downregulation in prostate cancer leads to oxidative stress and carcinogenesis. ${ }^{18}$ There are few studies on the immune function of SELENOP, and its potential immune mechanism is typically discussed in terms of proinflammatory immune cells and immune regulation of the liver. The loss of SELENOP may increase polarization of $\mathrm{M} 2$ macrophages and promote the occurrence of inflammatory tumors. ${ }^{19}$ SELENOP mRNA is upregulated in patients with chronic hepatitis C. It has also been shown to inhibit retinoic-acidinducible gene I (RIG-I)-mediated type I IFN response, resulting in persistent hepatitis $\mathrm{C}$ virus infection. ${ }^{20}$

Based on the above evidence, SELENOP may be related to tumorigenesis due to its Se transport function, antioxidant properties and immune function. Hence, the present study conducted the first pan-cancer analysis of SELENOP using multiple datasets and bioinformatics tools. The association between SELENOP expression levels and various factors, such as prognosis, genetic alteration, immune cells, tumor microenvironment and signaling pathways, was investigated in order to explore the multifaceted properties pan-cancer properties of SELENOP.

\section{Materials and Methods Collection of TCGA Pan-Cancer Data and Patient Selection}

The data used in this study were obtained from the TCGA database (https://portal.gdc.cancer.gov/). The TCGA database is a cancer genomics program of the National Cancer Institute (NCI) and the National Human Genome Research Institute, which includes genomic, epigenomic, transcriptional, and proteomic data for 33 tumor types. The data are widely used in cancer research and offer help to the diagnosis, treatment and prevention of cancer.

11,057 samples were downloaded by open-source software. These samples covered 33 different types of cancer, and comprised 10,327 tumor samples and 730 normal samples. Samples had received other adjuvant treatments were excluded. These data were used to analyze the expression pattern of SELENOP in 33 tumor types, the effect of SELENOP expression on survival, the association between SELENOP expression levels and tumor mutation burden (TMB), microsatellite instability (MSI), mismatch repair (MMR) gene expression, tumor microenvironment and signaling pathways in different cancers.

\section{Expression Analysis of SELENOP Based on Multiple Databases}

Based on mRNA expression data downloaded from the TCGA database, we analyzed the differences in SELENOP expression between tumor and normal tissues in 33 different types of cancer. Detailed information about 33 tumor types is presented in Table 1. Due to the absence or limited number of normal tissues in some cancers, we additively analyzed the expression differences in TCGA-based tumor tissues combined with GTEx normal tissues using the GEPIA2 (http://gepia2.cancer-pku.cn/) online tool. ${ }^{21}$

Based on the UALCAN portal (http://ualcan.path.uab. edu/analysis-prot.html), we validated six cancers at the protein level using the CPTAC dataset, including breast cancer, ovarian cancer, colon cancer, clear cell RCC 
Table I Summary of Detailed Information for 33 Tumor Types in TCGA Database

\begin{tabular}{|c|c|c|c|c|}
\hline Primary Disease Type & ID & Total $\mathbf{N}$ & Primary Tumor & Normal Tissue \\
\hline Adrenocortical cancer & $\mathrm{ACC}$ & 79 & 79 & 0 \\
\hline Bladder urothelial carcinoma & BLCA & 430 & 411 & 19 \\
\hline Breast invasive carcinoma & BRCA & 1217 & 1104 & 113 \\
\hline Cervical \& endocervical cancer & CESC & 309 & 306 & 3 \\
\hline Cholangiocarcinoma & $\mathrm{CHOL}$ & 45 & 36 & 9 \\
\hline Colon adenocarcinoma & COAD & 512 & 471 & 41 \\
\hline Diffuse large B-cell lymphoma & DLBC & 48 & 48 & 0 \\
\hline Esophageal carcinoma & ESCA & 173 & 162 & II \\
\hline Glioblastoma multiforme & GBM & 173 & 168 & 5 \\
\hline Head \& neck squamous cell carcinoma & HNSC & 546 & 502 & 44 \\
\hline Kidney chromophobe & $\mathrm{KICH}$ & 89 & 65 & 24 \\
\hline Kidney clear cell carcinoma & $\mathrm{KIRC}$ & 607 & 535 & 72 \\
\hline Kidney papillary cell carcinoma & KIRP & 321 & 289 & 32 \\
\hline Acute myeloid leukemia & LAML & $|5|$ & $|5|$ & 0 \\
\hline Brain lower grade glioma & LGG & 529 & 529 & 0 \\
\hline Liver hepatocellular carcinoma & LIHC & 424 & 374 & 50 \\
\hline Lung adenocarcinoma & LUAD & 585 & 526 & 59 \\
\hline Lung squamous cell carcinoma & LUSC & 550 & 501 & 49 \\
\hline Mesothelioma & MESO & 86 & 86 & 0 \\
\hline Ovarian serous cystadenocarcinoma & OV & 379 & 379 & 0 \\
\hline Pancreatic adenocarcinoma & PAAD & 182 & 178 & 4 \\
\hline Pheochromocytoma \& paraganglioma & PCPG & 186 & 183 & 3 \\
\hline Prostate adenocarcinoma & PRAD & 551 & 499 & 52 \\
\hline Rectum adenocarcinoma & READ & 177 & 167 & 10 \\
\hline Sarcoma & SARC & 265 & 263 & 2 \\
\hline Skin cutaneous melanoma & SKCM & 472 & 471 & I \\
\hline Stomach adenocarcinoma & STAD & 407 & 375 & 32 \\
\hline Testicular germ cell tumor & TGCT & 156 & 156 & 0 \\
\hline Thyroid carcinoma & THCA & 568 & 510 & 58 \\
\hline Thymoma & THYM & 121 & 119 & 2 \\
\hline Uterine corpus endometrioid carcinoma & UCEC & 583 & 548 & 35 \\
\hline Uterine carcinosarcoma & UCS & 56 & 56 & 0 \\
\hline Uveal melanoma & UVM & 80 & 80 & 0 \\
\hline
\end{tabular}

(Renal cell carcinoma), UCEC (uterine corpus endometrioid carcinoma) and LUAD (lung adenocarcinoma). ${ }^{22}$

\section{Survival Analysis of SELENOP in Pan-Cancer}

Survival data from TCGA dataset were used to analyze the relationship between SELENOP expression and prognosis in patients with different types of tumor by univariate methods. In order to comprehensively analyze the effect of SELENOP expression on tumor prognosis, three outcome indicators were included in our study, namely overall survival (OS), progression-free survival (PFS) and disease-specific survival (DSS). Meanwhile, the survival difference of
SELENOP low expression and high expression was explored by Kaplan-Meier analysis according to the above three indicators.

\section{Genetic Alteration Analysis of SELENOP}

Based on the cancer genomics data of cBioPortal (https://www.cbioportal.org/), we selected the mutation type and alteration frequency of TCGA pan-cancer studies for alteration analysis of SELENOP status in different tumor types. ${ }^{23}$ Next, four survival rates were used to describe the difference in survival with and without the SELENOP alteration, including OS, PFS, DSS and disease-free survival (DFS). 
Correlation of SELENOP Expression with TMB, MSI and MMR Genes Expression in Pan-Cancer

TMB is defined as the total number of somatic gene coding errors, base substitutions, and gene insertion or deletion errors detected per million bases, and is a potential biomarker that can predict the response to immunotherapy. ${ }^{24}$ MSI associated with DNA MMR defects is another significant biomarker for prognostic and therapeutic outcomes. MSI refers to the phenomenon that new microsatellite alleles appear at a microsatellite locus in tumor due to the insertion or deletion of repeated units compared with normal tissue. ${ }^{25}$ We calculated TMB scores by segmenting the total length of the exon using the Perl software. Similarly, MSI scores were calculated based on TCGA somatic mutation data in 33 tumor types. Next, we analyzed the association between SELENOP expression and these two biomarker scores.

MMR is helpful for the repair of DNA replication errors, and the loss of related gene functions can lead to the increased frequency of somatic mutations. ${ }^{26}$ The correlation between SELENOP expression and MMR genes (MLH1, MSH2, MSH6, PMS2 and EPCAM) was assessed in 33 tumor types.

\section{Immunity Correlation Analysis of SELENOP in Pan-Cancer}

The relationship between SELENOP expression and immune infiltration was conducted using the TIMER (http://cistrome.org/TIMER/), which is a web server for comprehensive analysis of tumor immune infiltration. ${ }^{27}$ SELENOP expression with the abundances of six immune infiltrates (B cells, CD4+ T cells, CD8+ T cells, Neutrophils, Macrophages and Dendritic cells) correlation analysis was performed. Moreover, the database can also quantify the effect of SELENOP expression on tumor purity.

In addition, we determined the correlation between cell markers of macrophage subtypes and SELENOP expression in 33 tumor types using the GEPIA. We selected the immune gene markers of tumor-associated macrophages (TAMs), M1 macrophages and M2 macrophages via the website of R\&DSystems (https://www.rndsystems.com/cn/ resources/cell-markers/immune-cells). These gene markers include CD80, CD86, HLA-G, CD36, IL-6, NOS2, TGF , STAT6 and $I L-10$.

Tumor cells and their surrounding immune cells, tumor fibroblasts and vascular endothelial cells constitute the tumor microenvironment and determine the development of tumors. In order to evaluate the association between SELENOP expression and the infiltration level of stromal and immune cells in different types of tumor, we first determined stromal and immune scores by using ESTIMATE algorithm to quantitatively evaluate the infiltration level of stromal and immune cells. Then, we analyzed the association of SELENOP expression with stromal and immune scores using the $\mathrm{R}$ software.

\section{PPI and Enrichment Analysis of SELENOP}

We used GeneMANIA website (http://www.genemania. org) to construct Protein-Protein Interaction (PPI) network, including physical interaction, co-localization, protein domain, co-expressed and genetic interaction connections between SELENOP and related genes. To determine the biological process of SELENOP in 33 tumor types, Gene Set Enrichment Analysis (GSEA) was performed. We downloaded Gene Ontology (GO) from the GSEA website (https://www.gsea-msigdb.org/gsea/down loads.jsp) and used R software for enrichment analysis.

\section{Statistical Analysis}

We used the Wilcoxon rank-sum test to analyze differences in SELENOP expression between normal and tumor tissues. Correlation analysis was conducted using Spearman correlation method, including correlation of SELENOP expression with TMB, MSI and MMR gene expression, immune cells and tumor microenvironment in pan-cancer.

In survival analysis, Kaplan-Meier method and Log rank test were used to compare the association between different expression levels of SELENOP and patient prognosis. The relationship between SELENOP expression and OS, DSS and PFS in cancer patients was analyzed by univariate Cox proportional hazard model. All results analysis and visualization were performed using $\mathrm{R}$ software (version 3.6.1) and $P<0.05$ were considered statistically significant.

\section{Results}

\section{Pan-Cancer SELENOP Expression Level}

First, the expression levels of SELENOP were analyzed in various blood cell types and non-tumor tissues by utilizing a combination of different datasets. Analysis of a combination of data from the Human Protein Atlas (HPA), GTEx and function annotation of the mammalian genome 5 
(FANTOM5) revealed that SELENOP expression levels showed tissue enhancement, with the highest expression in the liver, followed by the intestine (Figure S1A). Human brain SELENOP expression profile was determined based on a combination of data from GTEx and FANTOM5. SELENOP can be expressed in ten main brain regions (all consensus normalized expression values $>10$ ), showing low regional specificity (Figure S1B) . Because its corresponding protein is secreted into the blood, low cell type specificity was also present in 18 blood cell types and total peripheral blood mononuclear cells in the SELENOP expression analyzed based on the data from HPA/Monaco/Schmiedel (Figure S1C).

Next, we explored the expression patterns of SELENOP in TCGA pan-cancer data. The results showed that SELENOP expression was significantly higher in normal tissues, including BLCA (bladder urothelial carcinoma), BRCA (breast invasive carcinoma), $\mathrm{CHOL}$ (cholangiocarcinoma), COAD (colon adenocarcinoma), ESCA (esophageal carcinoma), HNSC (head and neck squamous cell carcinoma), KICH (kidney chromophobe), KIRC (kidney clear cell carcinoma), KIRP (kidney papillary cell carcinoma), LIHC (liver hepatocellular carcinoma), LUAD, LUSC (lung squamous cell carcinoma), PCPG (pheochromocytoma and paraganglioma), PRAD (prostate adenocarcinoma), READ (rectum adenocarcinoma), STAD (stomach adenocarcinoma), THCA (thyroid carcinoma) and UCEC, compared to that in the corresponding tumor tissues (Figure 1A). Considering the absence or a limited number of normal tissues in some cancers, the GEPIA2 was used to analyze SELENOP expression in several cancer types in the GTEx database. Similarly, a lower SELENOP expression was found in OV (ovarian serous cystadenocarcinoma), SKCM (skin cutaneous melanoma) and UCS (uterine carcinosarcoma) in the tumor groups (Figure 1B). However, SELENOP expression in the tumor tissues was significantly higher in DLBC (diffuse large B-cell lymphoma), LAML (acute myeloid leukemia) and LGG (brain lower grade glioma) than in normal tissues.

The expression pattern of $\mathrm{SeP}$ at a protein level was investigated in the CPTAC dataset. The results suggested that SeP expression in the normal tissues was significantly higher than that in the tumor tissues, including clear cell $\mathrm{RCC}$, breast cancer, ovarian cancer, colon cancer, LUAD and UCEC (Figure 1C).

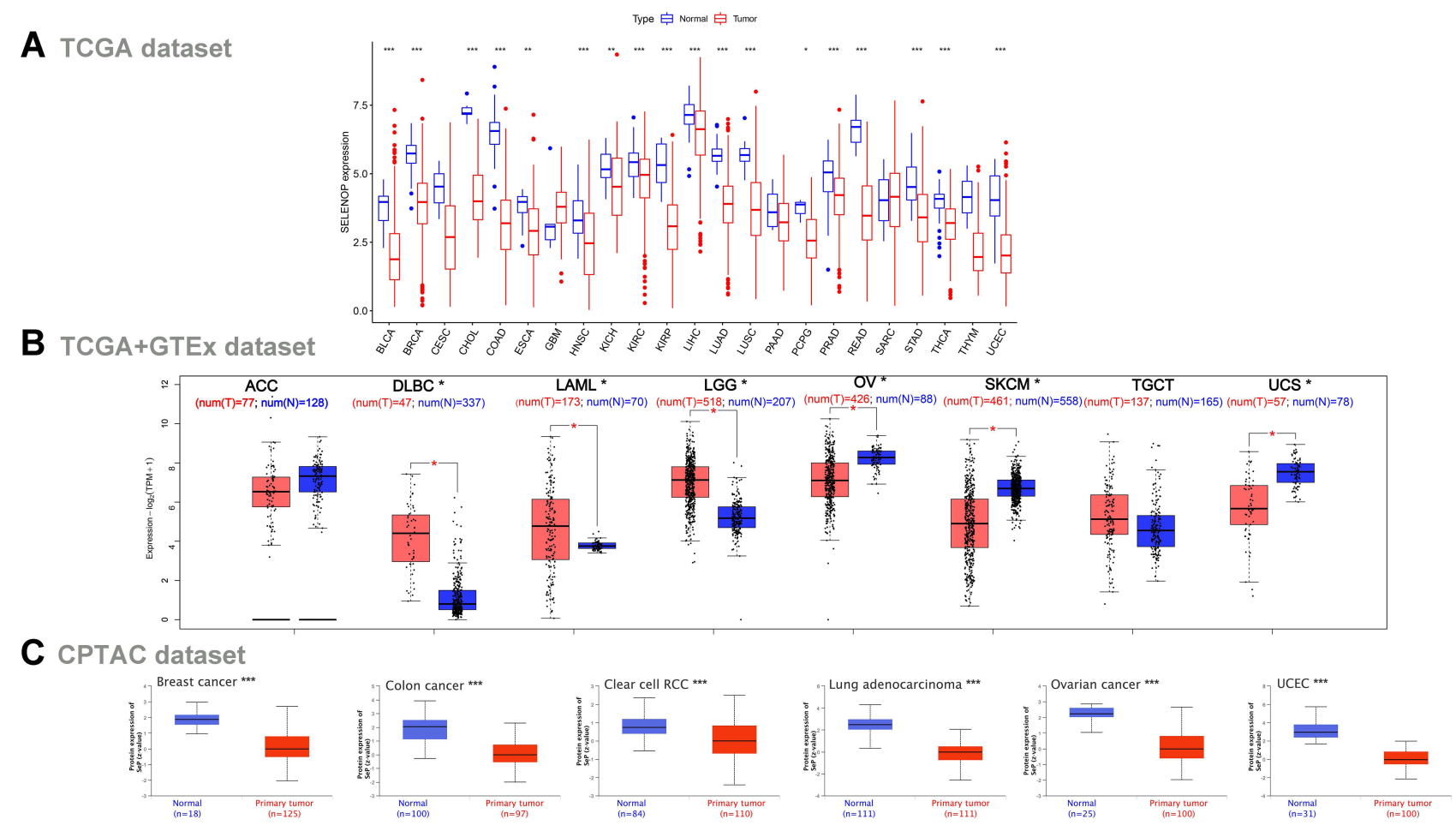

Figure I Expression level of SELENOP in different tumors. (A) SELENOP expression in 33 tumor types (based on TCGA database). (B) The corresponding normal tissues of the GTEx database were included as controls, compared with ACC, DLBC, LAML, LGG, OV, SKCM, TGCT, UCS tumor tissues in TCGA database. (C) The expression level of SeP total protein in 6 tumors (based on CPTAC dataset). $* P<0.05$, **P $<0.0$ I, $* * * P<0.00$ I.

Abbreviations: ACC, adrenocortical cancer; DLBC, diffuse large B-cell lymphoma; LAML, acute myeloid leukemia; LGG, brain lower grade glioma; OV, ovarian serous cystadenocarcinoma; SKCM, skin cutaneous melanoma; TGCT, testicular germ cell tumor; UCS, uterine carcinosarcoma. 


\section{Pan-Cancer Multifaceted Prognostic Potential and Genetic Alteration of SELENOP}

TCGA dataset was used to evaluate the pan-cancer correlation of SELENOP expression with prognosis values. The OS, PFS and DSS results were obtained using a Cox proportional-hazards model (Figure 2). Notably, the forest plots revealed that $S E L E N O P$ expression was significantly correlated with five cancer types, including ACC (adrenocortical cancer), KIRC, LGG, LUAD and UCEC. Among them, SELENOP expression had a protective role in the prognosis of three cancer types, including ACC (OS HR = $0.712, P=0.045$; PFS HR $=0.771, P=0.039)$, KIRC (OS $\mathrm{HR}=0.630, P<0.001 ;$ PFS HR $=0.669, P<0.001 ;$ DSS
$\mathrm{HR}=0.562, P<0.001)$, and LUAD $(\mathrm{OS} \mathrm{HR}=0.805, P<$ 0.001; PFS HR $=0.867, P=0.009$ ). In addition, SELENOP had a detrimental role in the other two cancer types, including LGG (OS HR $=1.303, P=0.003$; PFS $\mathrm{HR}=1.238, P=0.004$; DSS HR $=1.365, P<0.001)$ and UCEC $(\mathrm{OS} \mathrm{HR}=1.340, P=0.002$; PFS HR $=1.197, P=$ 0.016 ; DSS HR $=1.278, P=0.020$ ).

The KM curves for OS, PFS and DSS in patients with

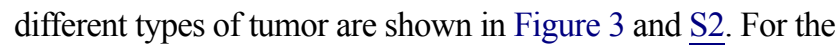
OS, highly expressed $S E L E N O P$ was linked to poor prognosis for LGG (Figure 3B; $P=0.001$ ) and UCEC (Figure 3D; $P=$ 0.002). A low expression level of SELENOP was related to a worse clinical outcome for KIRC (Figure 3A; $P<0.001$ ) and LUAD (Figure 3C; $P=0.005$ ). Moreover, PFS and DSS data analysis (Figure S2) revealed associations between high
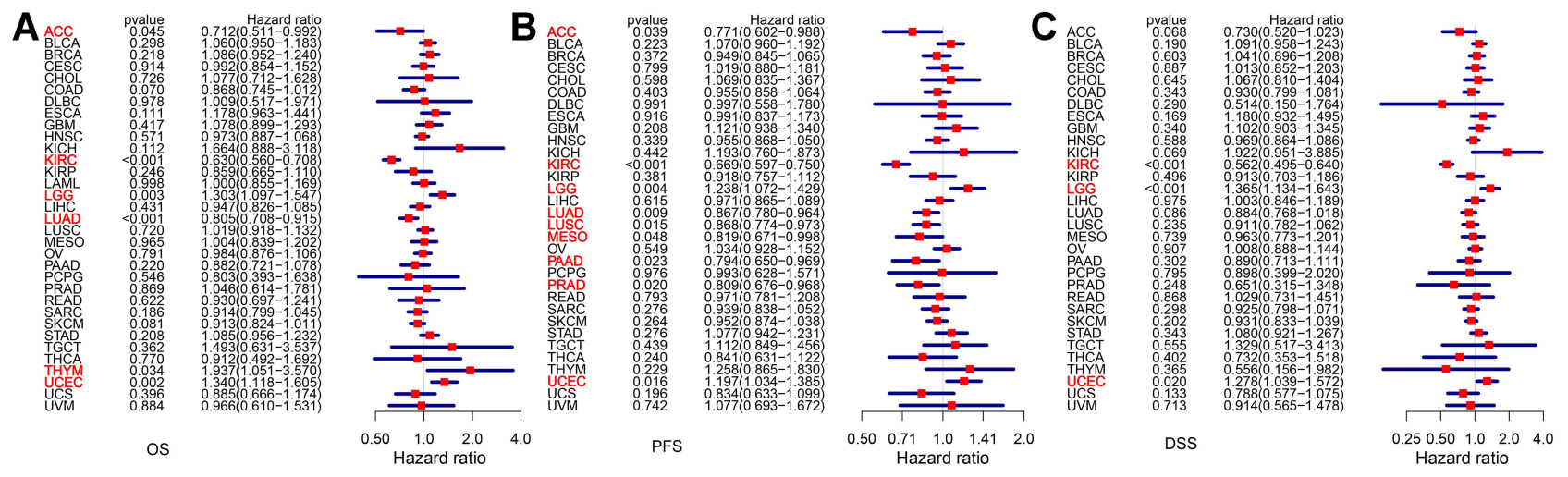

Figure 2 Association between SELENOP expression and OS, PFS and DSS. (A) Forest plot of OS associations in 33 types of tumor. (B) Forest plot of PFS associations in 33 types of tumor. (C) Forest plot of DSS associations in 33 types of tumor.

Abbreviations: OS, overall survival; PFS, progression-free survival; DSS, disease-specific survival.

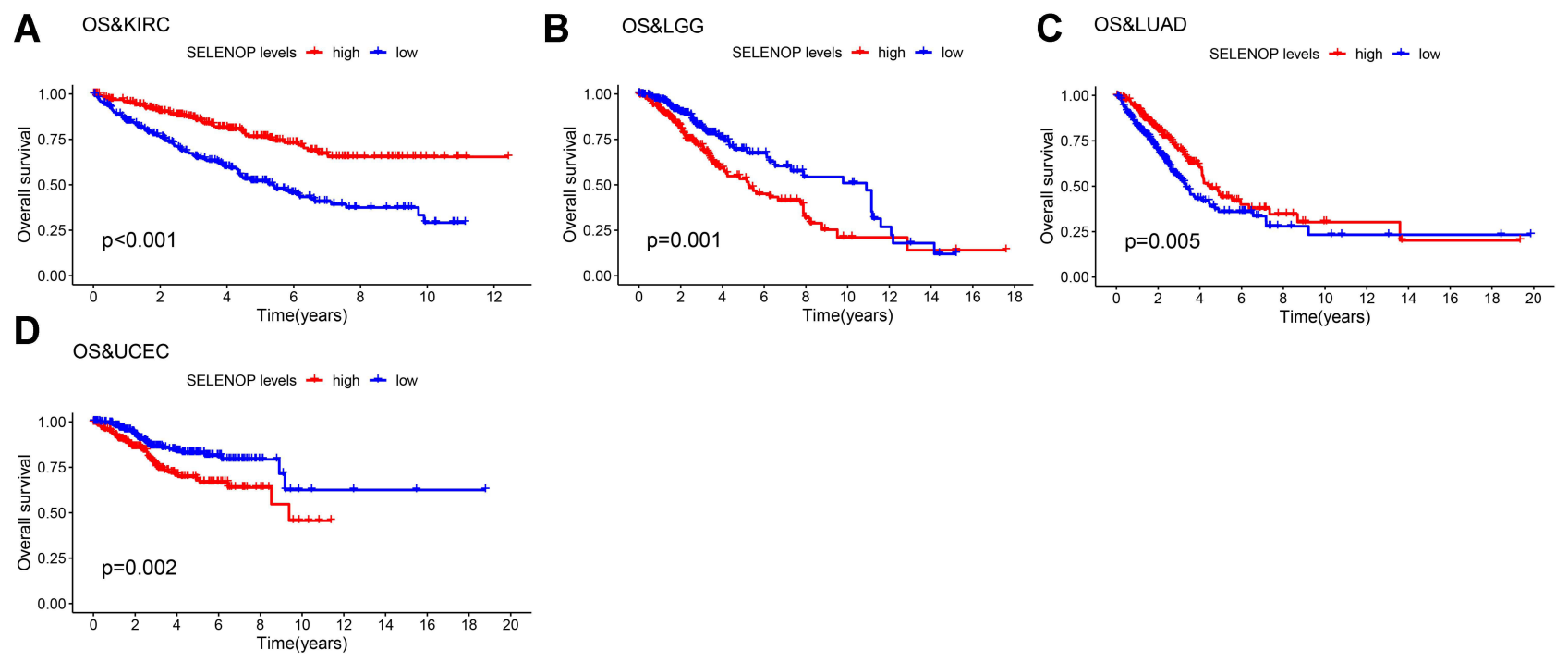

Figure 3 Kaplan-Meier analysis of the association between SELENOP expression and OS. (A) Kaplan-Meier plot of high and low SELENOP expression in KIRC patients. (B) and in LGG patients. (C) and in LUAD patients. (D) and in UCEC patients.

Abbreviations: KIRC, kidney clear cell carcinoma; LGG, brain lower grade glioma; LUAD, lung adenocarcinoma; UCEC, uterine corpus endometrioid carcinoma. 
SELENOP expression and poor prognosis for LGG and UCEC. However, SELENOP expression had the opposite relationship in KIRC. Analysis results for SELENOP in TCGA survival data confirmed its multifaceted prognostic potential that depends on the tumor type.

The genetic alteration of SELENOP was investigated in patients with different tumors using the cBioPortal database in order to determine if it plays a role in prognosis. The results suggested that amplification was the primary alteration type in most cancer cases, such as in LUAC, LUAD, ESCA and STAD (Figure 4A). In addition, SELENOP mutation frequencies were highest in SKCM, followed by UCEC. Next, we explored whether genetic alteration of SELENOP and clinical prognosis outcomes were related. Compared to the altered group of UCEC cases, the unaltered group was related to a better prognosis for DSS $(P=0.014)$ and PFS $(P=0.026)$, but not for $\operatorname{OS}(P=0.115)$ and DFS $(P=0.170$; Figure 4B-E).

\section{Relationships Between SELENOP Expression and MSI, TMB and MMR} Genes

The relationship between MSI, TMB and MMR genes and SELENOP expression was analyzed to further explore the role of SELENOP in tumorigenesis. There was a significant negative correlation with SELENOP expression and MSI in 12 tumor types, such as BRCA, $\mathrm{KICH}$, LUAD, OV, SKCM and STAD (Figure 5A). Except for LAML, which had a positive correlation with SELENOP expression and TMB, the other 14 cancer types, including BRCA, LGG, LUAD, PAAD (pancreatic adenocarcinoma), SKCM, STAD and UCEC, showed a significant negative correlation (Figure 5B). In addition, except in ESCA, GBM (glioblastoma multiforme) and LGG, SELENOP expression was positively correlated with MMR genes in most tumor types (Figure 5C).

\section{Relationships Between SELENOP Expression and Immune Infiltration and Tumor Microenvironment}

The activity pattern of immune cells affects tumor development and patient survival times. Therefore, immune infiltration analysis based on the TIMER database was performed to examine the relationship between SELENOP expression and infiltration levels in pancancer. Infiltration level was calculated using the score for the following six types of immune cells: B cells,
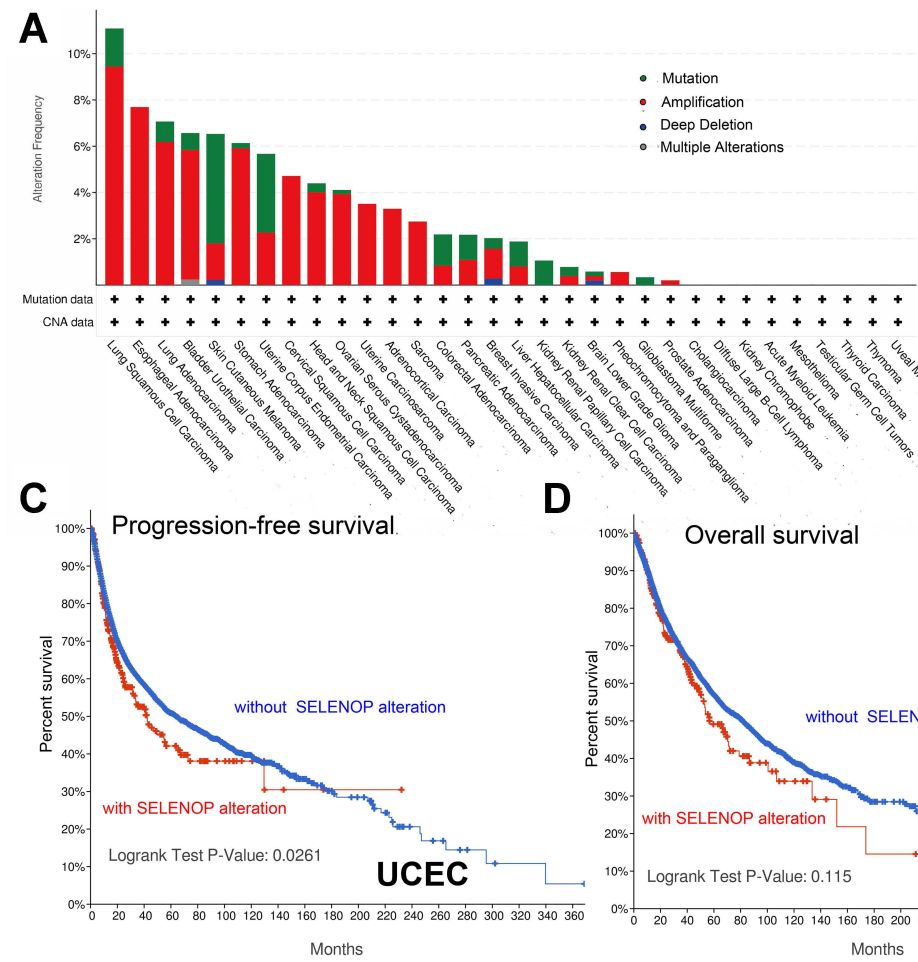

\section{B}

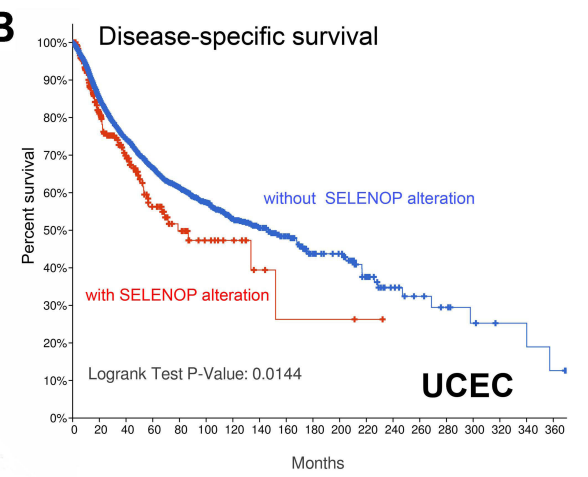

D
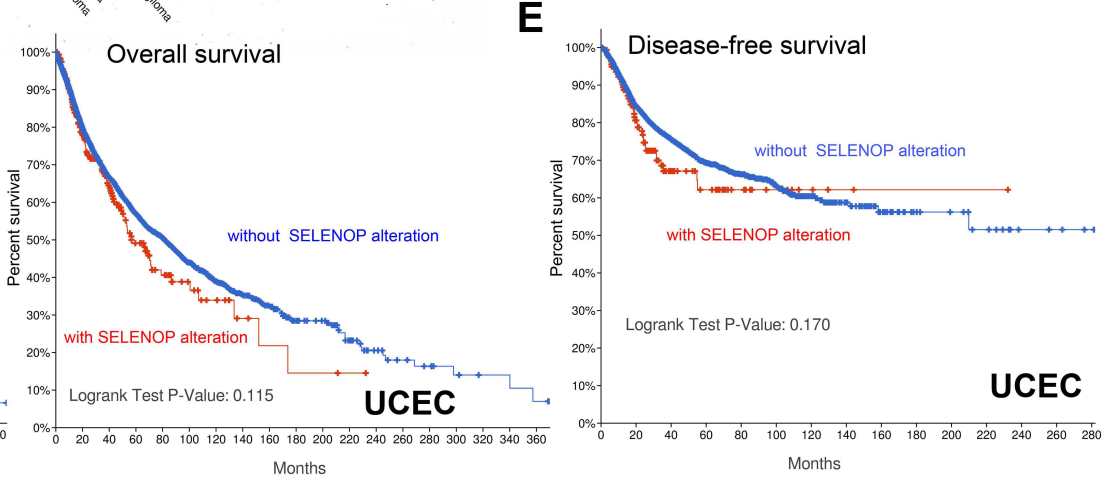

Figure 4 Mutation feature of SELENOP in different tumor types of TCGA. (A) The genetic alteration type and frequency of SELENOP in various tumors (based on the cBioPortal tool). (B) The potential correlation between mutation status and disease-specific survival of UCEC. (C) and progression-free survival of UCEC. (D) and overall survival of UCEC. (E) disease-free survival of UCEC.

Abbreviation: UCEC, uterine corpus endometrioid carcinoma. 


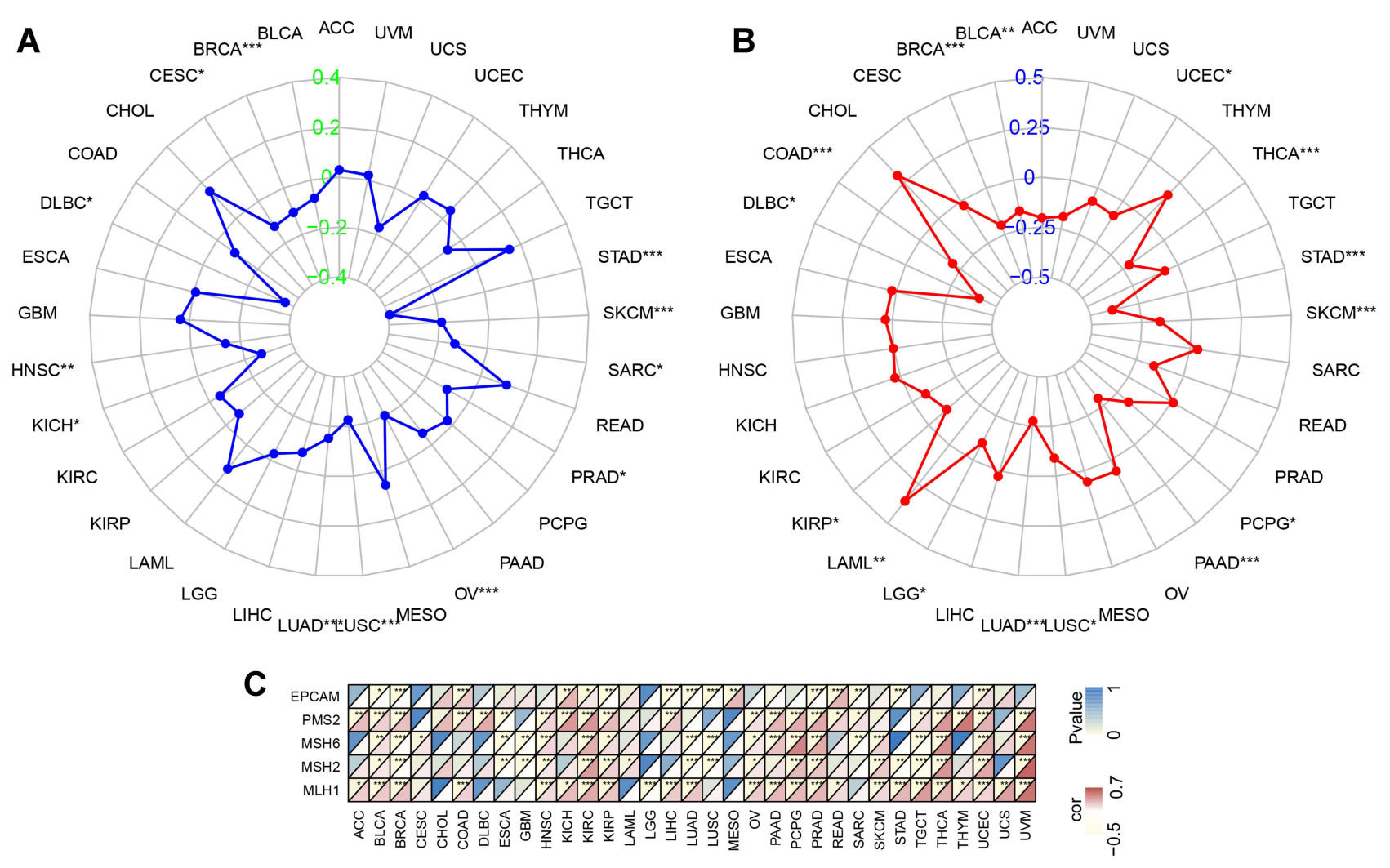

Figure 5 Associations between SELENOP expression and microsatellite instability (MSI), tumor mutational burden (TMB) and mismatch repair (MMR) in 33 tumor types. (A) Results of correlation analysis between SELENOP expression in 33 tumor types and MSI. (B) and TMB. The P-value is supplied. The correlation coefficient values of +0.4 and -0.4 are marked. (C) and five MMR genes. The top left triangle represents the $P$-value, and the bottom right triangle represents the correlation coefficient. $* P<0.05$, $* * P<0.01$ and $* * * P<0.001$.

CD4+ T cells, CD8+ T cells, neutrophils, macrophages and dendritic cells.

There were four types of cancer whose SELENOP expression was associated with the infiltration levels of all six immune cell types (Figure 6). SELENOP expression had a significant negative correlation with tumor purity, except in KIRC $(\mathrm{r}=-0.034, P<0.0001)$. The results also suggested that increased infiltration of six immune cell types was associated with increased SELENOP expression in the following cancers: KIRC, LGG, LUAD and SKCM. However, SELENOP expression had a strong positive correlation with macrophage infiltration levels in four tumor types $(\mathrm{r}>0.4, P<$ 0.0001). To further examine the relationship between macrophage infiltration and SELENOP, the correlation analysis between cell markers of macrophage subtypes and SELENOP expression was conducted in GEPIA. Immune marker genes for macrophage subtypes included tumor-associated macrophages (CD80, CD86 and $H L A-G), \quad \mathrm{M} 1$ macrophages (CD36, IL-6 and NOS2), and M2 macrophages (TGF 3 , STAT6 and $I L$ -
10). The results revealed that SELENOP expression was positively correlated with most macrophage immune marker genes in pan-cancer (Table 2). However, there was no evidence to support the regulation of macrophage polarization via SELENOP expression in any type of cancer.

Furthermore, the effect of pan-cancer SELENOP expression using the $\mathrm{R}$ package estimate was determined in the immune microenvironment. SELENOP expression had a significant positive correlation with stromal and immune scores in TGCT, SKCM, SARC, PCPG, KIRP, PAAD and GBM (Figure 7). The six tumors with the highest correlation coefficients between SELENOP expression and stromal scores were TGCT $(\mathrm{R}=0.61)$, SKCM ( $\mathrm{R}$ $=0.55)$, SARC (sarcoma) $(\mathrm{R}=0.52)$, PCPG $(\mathrm{R}=0.51)$, KIRP $(\mathrm{R}=0.46)$ and PAAD $(\mathrm{R}=0.43)$. The top six tumors whose SELENOP expression was most significantly positively correlated with immune scores were SARC $(R=0.43)$, SKCM $(R=0.40)$, PAAD $(R=0.40)$, GBM $(\mathrm{R}=0.37)$, PCPG $(\mathrm{R}=0.37)$ and $\mathrm{KIRP}(\mathrm{R}=0.32)$ (all $P<0.05$ ). 


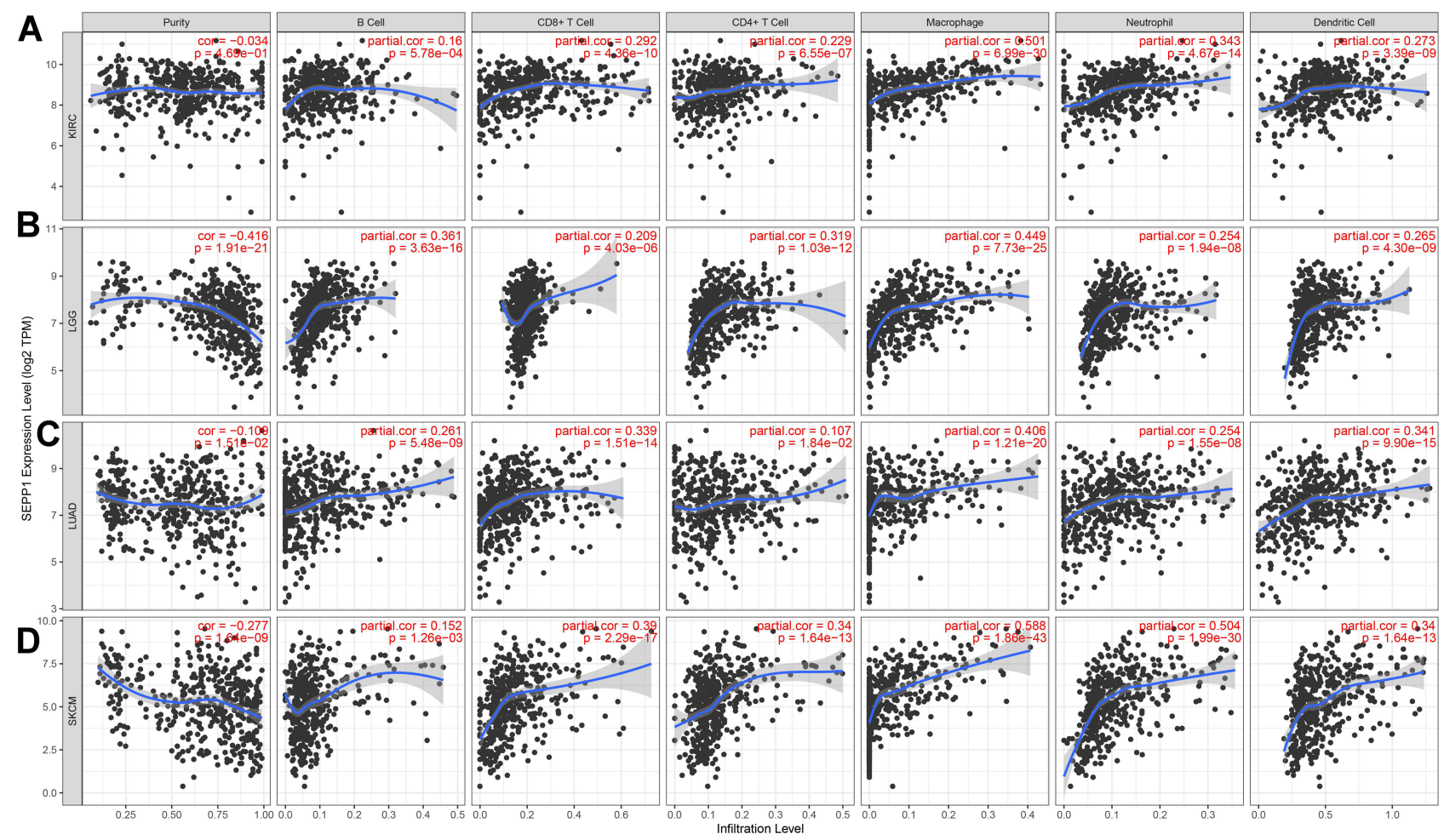

Figure 6 Associations between SELENOP expression and tumor immune infiltration. (A) Correlation analysis between expression levels of SELENOP and immune cell infiltration in KIRC. (B) and in LGG. (C) and in LUAD. (D) and in SKCM.

Abbreviations: KIRC, kidney clear cell carcinoma; LGG, brain lower grade glioma; LUAD, lung adenocarcinoma; SKCM, skin cutaneous melanoma.

\section{SELENOP PPI Network and GSEA in Pan-Cancer}

To further investigate the roles for SELENOP in the development of 33 tumor types, a PPI network analysis and GSEA of SELENOP were conducted. Using the GeneMANIA online tool, a strong physical interaction was revealed between SELENOP and mesenchyme homeobox 2 (MEOX2; Figure 8). MEOX2 is expressed in vascular endothelial cells and it inhibits their proliferation. $^{28}$ This potential function is consistent with the pathway enrichment analysis in LGG and UCEC. A common feature of the GO enrichment term indicated that SELENOP negatively regulated vascular endothelial cell proliferation in LGG and UCEC (Figure 9A). Similarly, SELENOP expression was negatively correlated with cell proliferation, differentiation, and adhesion in KIRC, LUAD, STAD, BRCA, SARC and THCA, including epidermal cell differentiation, keratinization and epidermis development (Figure 9B and S3A). In contrast, in COAD, PCPG, SKCM, OV, PAAD and UVM, the process of upregulation was related to the immune function, including $\mathrm{T}$ cell activation, $\mathrm{B}$ cellmediated immunity, adaptive immune response and immune response regulation cell surface receptor signaling pathways (Figure 9C and $\underline{\mathrm{S} 3 \mathrm{~B}}$ ).

\section{Discussion}

The protective effect of selenoprotein family is usually attributed to its antioxidant properties in tumorigenesis. However, little research has been done on selenoproteins in tumor development, such as SELENOP. Due to the complexity of the mechanism, the relationship between selenoproteins and cancer should be analyzed from multiple perspectives, including DNA stability, immune responses and related signaling pathways. ${ }^{29-31}$ Thus, the present study attempted to explore the pan-cancer role of SELENOP in terms of tissue specificity, immune microenvironment and signaling pathways.

After intestinal absorption, various Se compounds reach the liver and are incorporated into selenoproteins, including SeP (Figure 10). ${ }^{32}$ Plasma SeP serves as a transport protein to supply Se to target tissues, such as the brain, kidney and testis. Many studies have revealed that SeP uptake in the brain and testis is mediated by Lrp8 (apolipoprotein E receptor 2, ApoER2), a member of the low-density lipoprotein receptor-related protein 


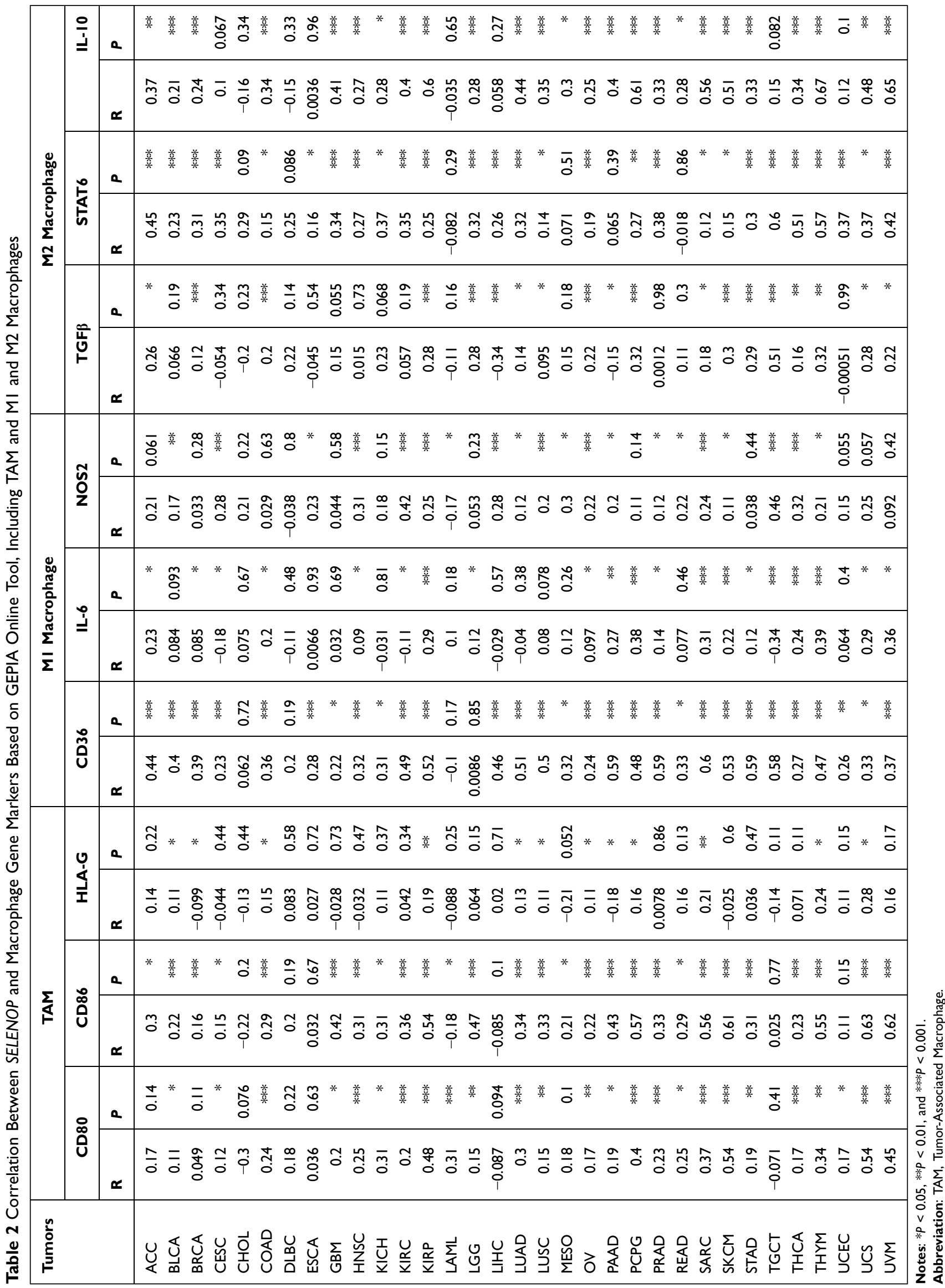



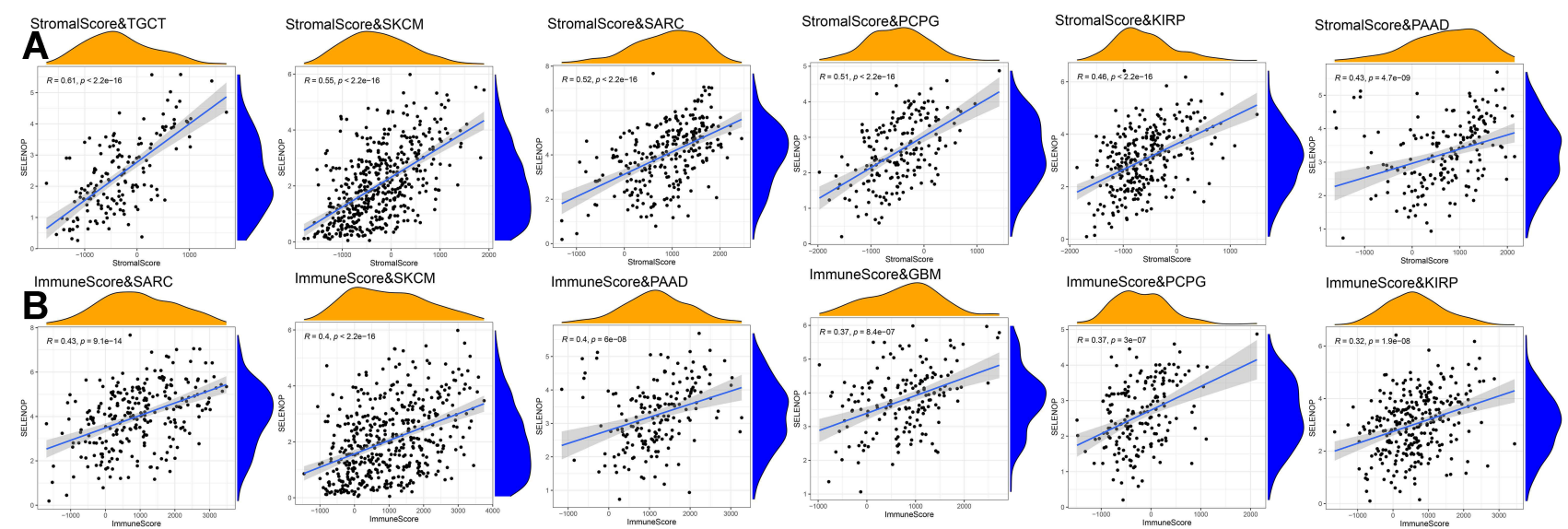

Figure 7 Six tumors with the highest correlation coefficients between SELENOP expression and the tumor microenvironment. (A) Correlation between SELENOP and stromal scores in TGCT, SKCM, SARC, PCPG, KIRP and PAAD. (B) Correlation between SELENOP and immune scores in SARC, SKCM, PAAD, GBM, PCPG and KIRP. Abbreviations: TGCT, testicular germ cell tumor; SKCM, skin cutaneous melanoma; SARC, sarcoma; PCPG, pheochromocytoma and paraganglioma; KIRP, kidney papillary cell carcinoma; PAAD, pancreatic adenocarcinoma; GBM, glioblastoma multiforme.

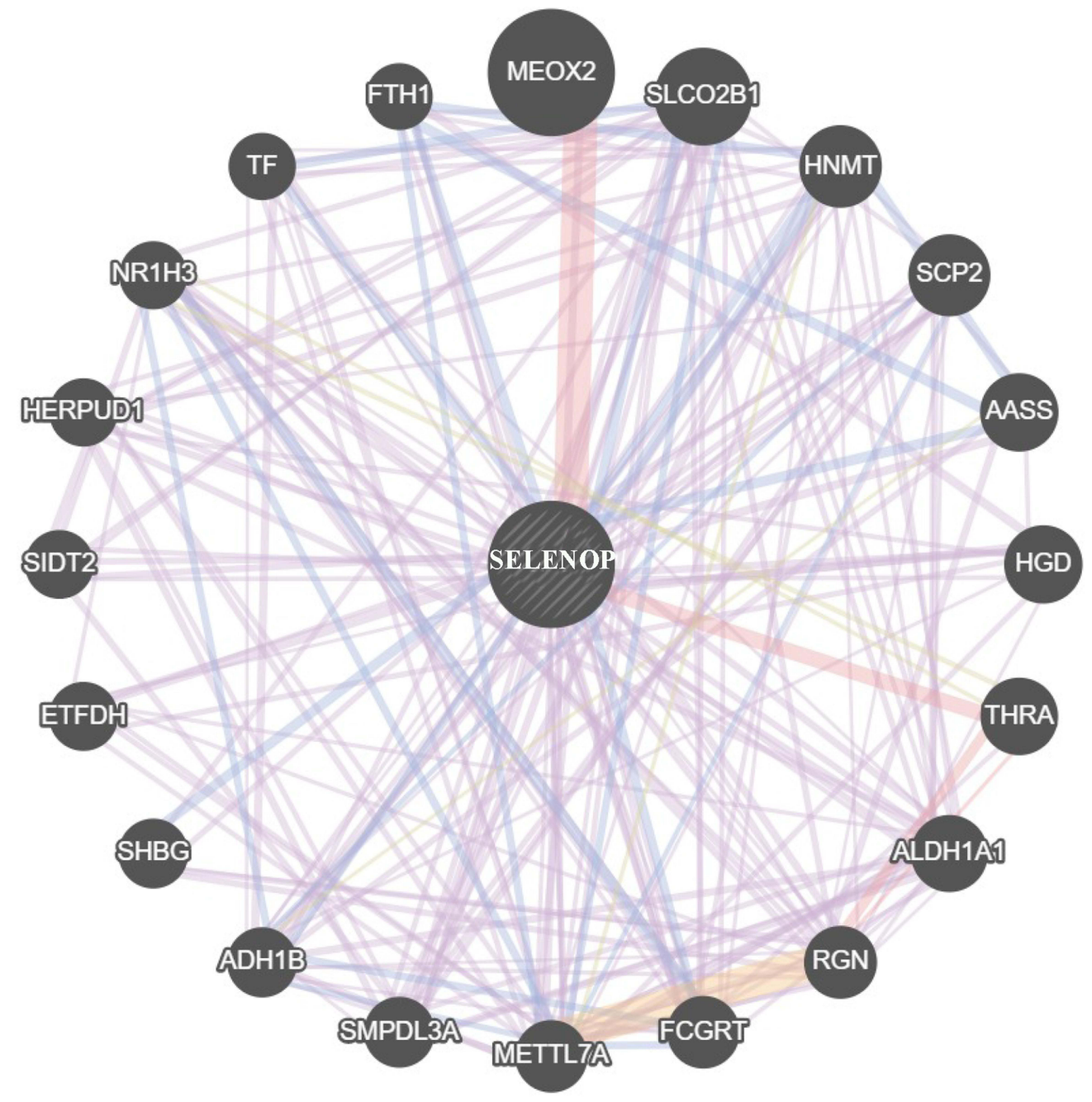

\section{Networks}

- Physical Interactions

Co-expression

Predicted

Co-localization

- Pathway

- Genetic Interactions

- Shared protein domains

Figure 8 PPI network for SELENOP based on GeneMANIA online tool. Different colors of the network edge indicate the bioinformatics methods applied: physical interaction, co-expression, predicted, colocalization, pathway, genetic interaction, and shared protein domains.

Abbreviation: PPI, protein-protein interaction. 
A
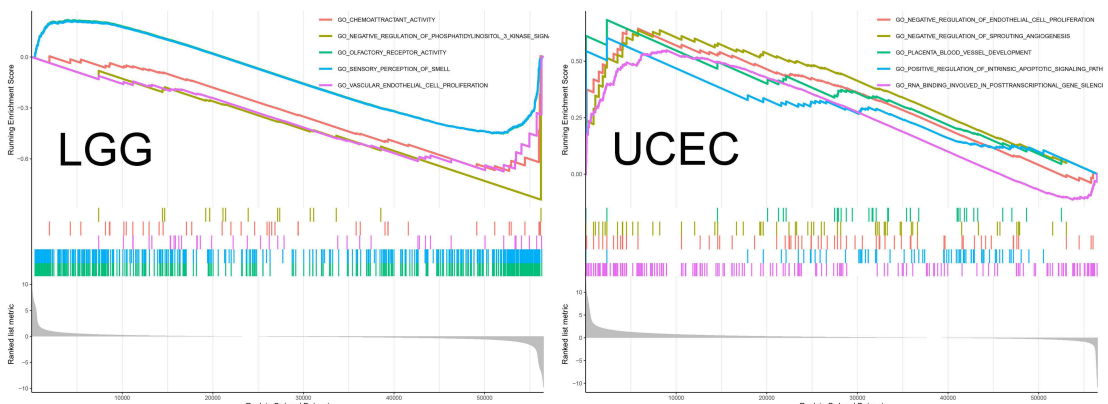

B
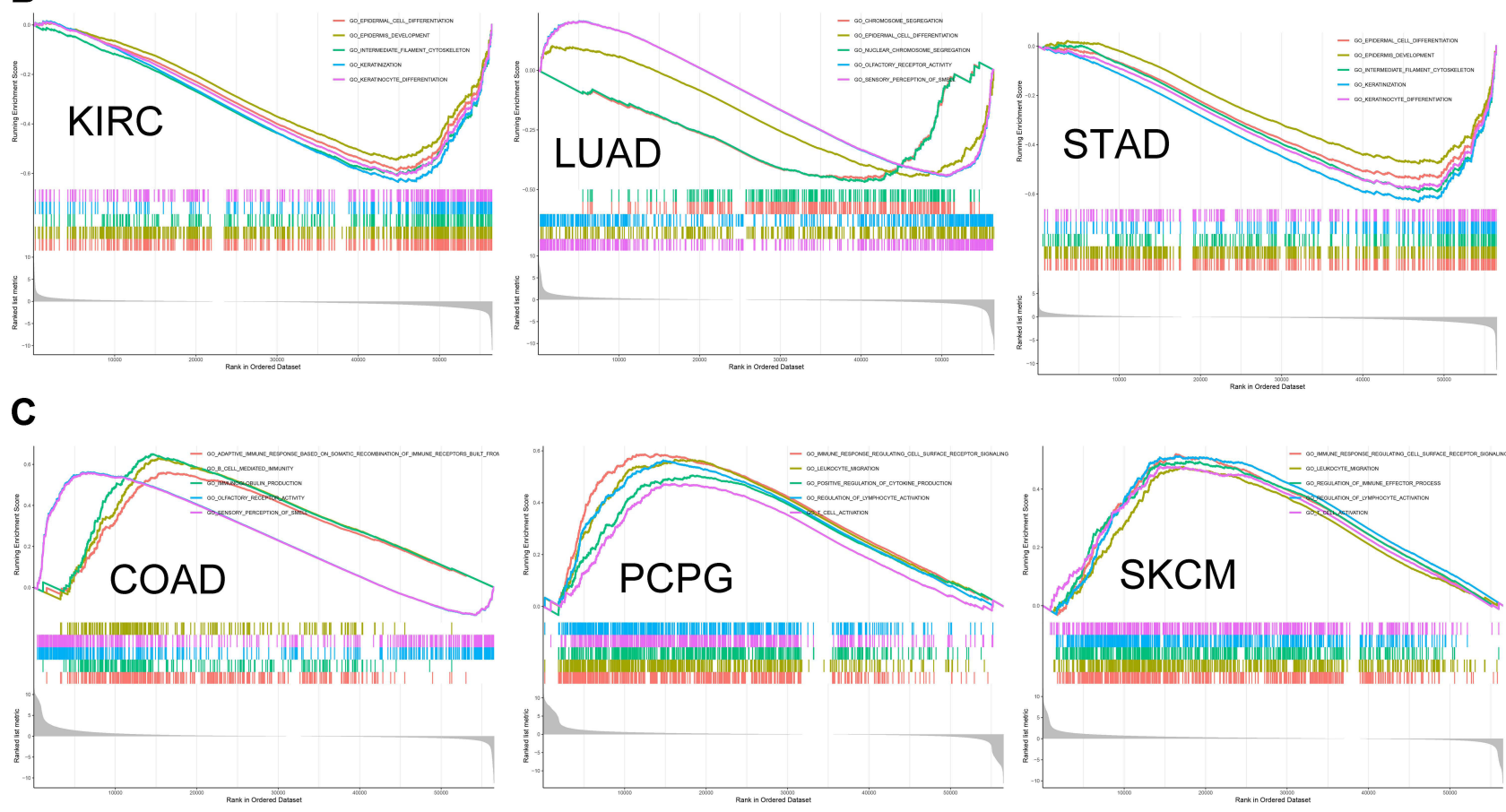

Figure 9 GO functional annotation of GSEA of SELENOP in various tumor types. (A) A common feature in LGG and UCEC was that SELENOP negatively regulated vascular endothelial cell proliferation. (B) SELENOP expression was negatively correlated with epidermal cell differentiation, keratinization and epidermis development in KIRC, LUAD and STAD. (C) In COAD, PCPG and SKCM, the process of upregulation was related to immune function, including T cell activation, B cell mediated immunity, adaptive immune response and immune response regulation cell surface receptor signaling pathways.

Abbreviations: COAD, colon adenocarcinoma; STAD, stomach adenocarcinoma; KIRC, kidney clear cell carcinoma; LGG, brain lower grade glioma; LUAD, lung adenocarcinoma; SKCM, skin cutaneous melanoma; UCEC, uterine corpus endometrioid carcinoma; PCPG, pheochromocytoma and paraganglioma.

family. ${ }^{33,34}$ In addition to Lrp8, SeP can also bind the Lrp2 (megalin) receptor in the kidney. ${ }^{35}$ Some tissues that express SELENOP but do not express $L r p 8$ and $L r p 2$ may exert the physiological effects of SELENOP through mechanisms that remain unclear.

Due to its transport function, SELENOP expression was decreased in many cancer tissues compared to normal tissues, such as in $\mathrm{COAD}$, $\mathrm{KICH}$, KIRC, KIRP, LIHC, LUAD, PRAD, SKCM, THCA and UCEC (Figure 1A). In contrast, SELENOP expression was upregulated in tumor tissues, including DLBC, LAML and LGG (Figure 1B). SELENOP expression downregulation has been demonstrated in several types of tumor, including hepatocellular carcinomas, gastric adenocarcinomas, colorectal cancer, non-small cell lung cancer, renal cancer and prostate cancer. ${ }^{17,36-40}$

Survival analysis in TCGA database showed that SELENOP expression was associated with a better prognosis in ACC, KIRC, LUAD and PRAD, and a poorer prognosis in LGG and UCEC (Figure 2). Higher alteration frequency of SELENOP was present in SKCM and UCEC compared to other cancers (Figure 4A). In addition, the association between SELENOP expression and prognosis may be related to mutation in UCEC, which has a detrimental impact on prognosis (Figure 4B-C). 
It may not be surprising that the absence of SELENOP's antioxidant protection promotes the development of some cancers. ${ }^{14,18}$ However, it cannot be ruled out that the relationship between $\mathrm{SeP}$ and the two receptors (Lrp8 and Lrp2) may regulate the downstream oncogenic signaling pathway. ${ }^{41}$ For example, apoER2 (Lrp8) knockdown of the downstream component of Reelin (a key regulator of neuronal migration) inhibits cell migration and invasion in pancreatic cancer. ${ }^{42}$ It is worth noting that SELENOP plays an important role in the brain. SELENOP mRNA is expressed in neurons and glial cells. ${ }^{43}$ In addition, SeP and apoER2 may supply Se to the brain at the blood-brain barrier. ${ }^{34}$ Most researchers have focused on SELENOP and neurodegenerative diseases, and there has been no investigation of the relationship between SELENOP and brain cancer. ${ }^{44,45}$ The present study found that SELENOP may be a risk factor in LGG, but the SeP-apoER2 interaction in tumorigenesis remains unclear.

SELENOP can also affect immune cell recruitment. Se deficiency has been shown to weaken the immune response of tumor cells. ${ }^{46}$ This condition is usually accompanied by changes in cytokines, such as $C O X-2, T N F-\alpha$, and $T G F-\beta .^{47}$ Likewise, several studies have confirmed that certain cytokines are regulated by SELENOP, including $T N F-\alpha$, hepatocyte growth factor, vascular endothelial growth factor (VEGF), and $I L-6 .{ }^{48,49}$ Although the specific mechanism remains unclear, Bosschaerts et al have reported that $I L-10$ promotes SELENOP expression. ${ }^{50}$ In studies related to the inflammatory response of SELENOP, it was highly expressed in alternatively activated M2 macrophage phenotype and involved in the conversion from classical M1 to M2 macrophage and in colitisassociated cancer. The tumorigenesis promotion may have occurred due to macrophage polarization in SELENOP heterozygous mice. ${ }^{19,50}$

The present results showed that SELENOP is associated with the infiltration level of six immune cell types, in which SELENOP had a strong correlation with macrophages in some cancer types (Figure 6). This may have been caused by the heterogeneity of immune cell recruitment in the tumor microenvironment. The relationship between SELENOP expression and immune marker genes of macrophage subtypes, such as $C D 80, C D 86$, HLA-G, CD36, IL-6, NOS2, TGF $\beta$, STAT6 and IL-10, was further analyzed (Table 2). Macrophage polarization was not present in 33 tumor types. Therefore, the oncogenic effect of SELENOP and macrophage polarization may occur in inflammatory tumorigenesis, while in most other tumors, SELENOP may exhibit anti-carcinogenic properties. ${ }^{51}$

The potential association between SELENOP and immune function was verified using pathway enrichment analysis in COAD, PCPG, SKCM, OV, PAAD and UVM (Figure $9 \mathrm{C}$ and $\underline{\mathrm{S} 3 \mathrm{~B}}$ ). Speckmann et al have reported that SELENOP secreted in the small intestine was involved in regulating the activation and differentiation of immune cells and maintained the local immune defense in the intestine. ${ }^{52}$ In addition, SELENOP may regulate cell proliferation, migration, adhesion and angiogenesis in some cancers (Figure 9B and $\underline{\mathrm{S} 3 \mathrm{~A}}$ ). Selenoproteins can inhibit tumor cell metastasis. ${ }^{7}$ For example, GPX2 and GPX3 negatively regulated cell migration and invasion, while TXNRD1 knockdown reduced VEGF and thus inhibited angiogenesis. $^{53-56}$

The present study represented the first pan-cancer analysis of SELENOP. Study results indicated a close relationship between SELENOP and tumorigenesis using

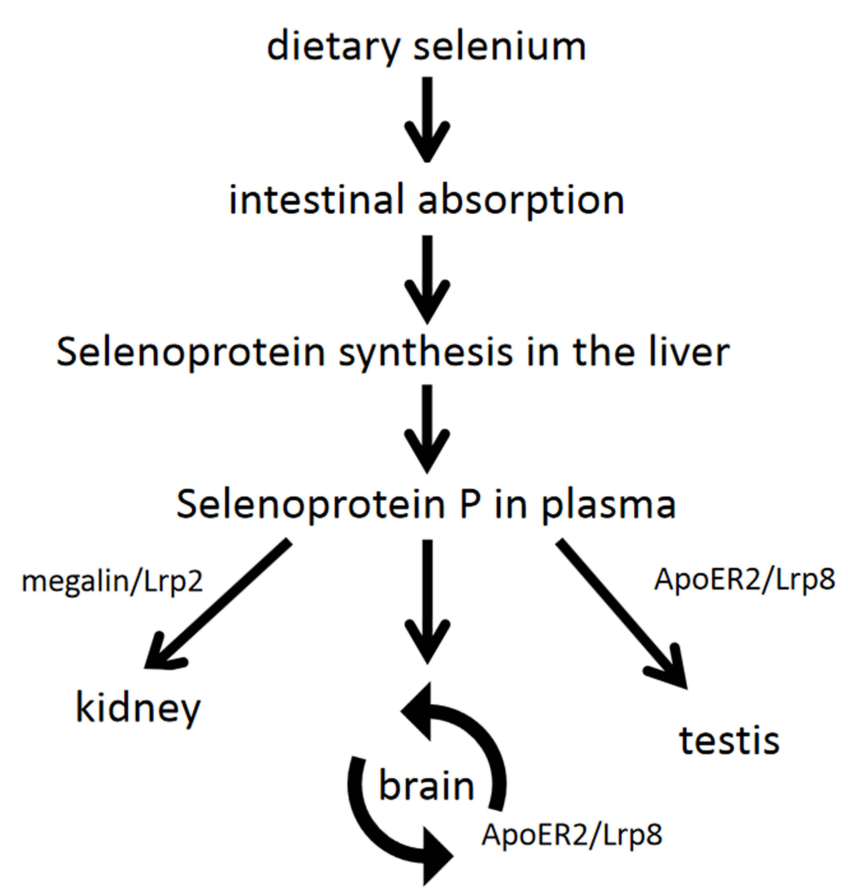

Figure 10 Summary of the transport function of SeP. Dietary Se is absorbed through the small intestine, and various Se compounds synthesize selenoprotein in the liver via circulation of portal vein, including SeP. Plasma SeP preferentially transports Se to various target tissues, such as the brain, kidney and testis. SeP transport in the brain is complicated because Se passes through multiple cell membranes before eventually reaching neurons. The neuronal SeP synthesis and ApoER2/Lrp8-mediated SeP reuptake in the brain are called the SeP cycle. Testicular function is also dependent on ApoER2/Lrp8-mediated SeP uptake. Megalin/Lrp2 is expressed in renal tubular epithelium and is involved in the reabsorption of $\mathrm{SeP}$ from glomerular filtrate.

Abbreviations: SeP, Selenoprotein P; Se, Selenium; ApoER2, apolipoprotein E receptor 2 . 
tissue-specific, immune function and pathway enrichment analyses. SELENOP expression was also shown to be downregulated and associated with a more favorable prognosis in most tumor tissues. A series of analyses indicated that SELENOP had anti-carcinogenic properties and a positive prognostic potential in most cancer types. In addition to SELENOP's antioxidant properties, the present study focused on its the multifaceted pan-cancer properties in immunity, cell proliferation, differentiation, invasion and angiogenesis. Although the current exploration of pan-cancer SELENOP mRNA was performed using bioinformatics, further experimental studies should focus on the specific SELENOP function in each cancer type. The present study had some limitations. First, it almost completely relied on mRNA levels. Second, the relationship among Se, SeP, SELENOP mRNA and immune status in cancers requires further study. Besides, whether the specific receptors and both immune and signaling pathways of SELENOP can have multiple effects in different cancer types remains an open question.

\section{Conclusion}

To the best of our knowledge, this is the first pan-cancer analysis of SELENOP to investigate its characteristics in tissue-specific expression, survival prognosis, TMB, MSI, immune function and pathway enrichment. Based on the present study results, SELENOP may be tissue-specific in cancers, such as the brain, kidney, lung, gastrointestinal and reproductive system tumors. In addition to its known antioxidant activity, the molecular mechanism of SELENOP in tumor immunity and metastasis requires a further investigation.

\section{Data Sharing Statement}

The data supporting the results of the study are available from the TCGA database (https://portal.gdc.cancer.gov/).

\section{Disclosure}

The authors declare that they have no conflict of interest.

\section{References}

1. Lu J, Holmgren A. Selenoproteins. J Biol Chem. 2009;284 (2):723-727.

2. Papp LV, Lu J, Holmgren A, Khanna KK. From selenium to selenoproteins: synthesis, identity, and their role in human health. Antioxid Redox Signal. 2007;9(7):775-806.
3. Kang D, Lee J, Wu C, et al. The role of selenium metabolism and selenoproteins in cartilage homeostasis and arthropathies. Exp Mol Med. 2020;52(8):1198-1208.

4. Burk RF, Hill KE, Motley AK. Selenoprotein metabolism and function: evidence for more than one function for selenoprotein P. $J$ Nutr. 2003;133(5):1517S-1520S.

5. Hatfield DL, Berry MJ, Gladyshev VN. Selenium: Its Molecular Biology and Role in Human Health. Springer Science \& Business Media; 2011.

6. Rayman MP. Selenium in cancer prevention: a review of the evidence and mechanism of action. Proc Nutr Soc. 2005;64(4):527-542.

7. Kuršvietienė L, Mongirdienè A, Bernatonienė J, Šulinskienė J, Stanevičienė I. Selenium anticancer properties and impact on cellular redox status. Antioxidants (Basel). 2020;9:1.

8. Amaral AFS, Cantor KP, Silverman DT, Malats N. Selenium and bladder cancer risk: a meta-analysis. Cancer Epidemiol Biomark Prev. 2010;19(9):2407-2415.

9. Glattre E, Thomassen Y, Thoresen SO, et al. Prediagnostic serum selenium in a case-control study of thyroid cancer. Int $J$ Epidemiol. 1989;18(1):45-49.

10. Wei W-Q, Abnet CC, Qiao Y-L, et al. Prospective study of serum selenium concentrations and esophageal and gastric cardia cancer, heart disease, stroke, and total death. Am J Clin Nutr. 2004;79(1):80-85.

11. Navarro-Alarcon M, Cabrera-Vique C. Selenium in food and the human body: a review. Sci Total Environ. 2008;400(1-3):115-141.

12. Wu W, Li D, Feng X, et al. A pan-cancer study of selenoprotein genes as promising targets for cancer therapy. BMC Med Genomics. 2021;14(1):78.

13. Stolwijk JM, Garje R, Sieren JC, Buettner GR, Zakharia Y. Understanding the redox biology of selenium in the search of targeted cancer therapies. Antioxidants (Basel). 2020;9:5.

14. Short SP, Whitten-Barrett C, Williams CS. Selenoprotein P in colitis-associated carcinoma. Mol Cell Oncol. 2016;3(3):e1075094.

15. Hill KE, $\mathrm{Wu} \mathrm{S}$, Motley AK, et al. Production of selenoprotein P (Sepp1) by hepatocytes is central to selenium homeostasis. $J$ Biol Chem. 2012;287(48):40414-40424.

16. Schweizer U, Streckfuss F, Pelt P, et al. Hepatically derived selenoprotein $\mathrm{P}$ is a key factor for kidney but not for brain selenium supply. Biochem J. 2005;386(Pt 2):221-226.

17. Gresner P, Gromadzinska J, Jablonska E, Kaczmarski J, Wasowicz W. Expression of selenoprotein-coding genes SEPP1, SEP15 and hGPX1 in non-small cell lung cancer. Lung Cancer. 2009;65(1):34-40.

18. Gonzalez-Moreno O, Boque N, Redrado M, et al. Selenoprotein-P is down-regulated in prostate cancer, which results in lack of protection against oxidative damage. Prostate. 2011;71(8):824-834.

19. Barrett CW, Reddy VK, Short SP, et al. Selenoprotein P influences colitis-induced tumorigenesis by mediating stemness and oxidative damage. J Clin Invest. 2015;125(7):2646-2660.

20. Murai K, Honda M, Shirasaki T, et al. Induction of selenoprotein $\mathrm{P}$ mRNA during hepatitis $\mathrm{C}$ virus infection inhibits RIG-I-mediated antiviral immunity. Cell Host Microbe. 2019;25(4):588-601.e587.

21. Tang Z, Kang B, Li C, Chen T, Zhang Z. GEPIA2: an enhanced web server for large-scale expression profiling and interactive analysis. Nucleic Acids Res. 2019;47(W1):W556-W560.

22. Chen F, Chandrashekar DS, Varambally S, Creighton CJ. Pan-cancer molecular subtypes revealed by mass-spectrometry-based proteomic characterization of more than 500 human cancers. Nat Commun. 2019;10(1):5679.

23. Gao J, Aksoy BA, Dogrusoz U, et al. Integrative analysis of complex cancer genomics and clinical profiles using the cBioPortal. Sci Signal. 2013;6(269):p11.

24. Yarchoan M, Hopkins A, Jaffee EM. Tumor mutational burden and response rate to PD-1 inhibition. $N$ Engl J Med. 2017;377 (25):2500-2501.

25. Li K, Luo H, Huang L, Luo H, Zhu X. Microsatellite instability: a review of what the oncologist should know. Cancer Cell Int. 2020;20:16. 
26. Dan H, Zhang S, Zhou Y, Guan Q. DNA methyltransferase inhibitors: catalysts for antitumour immune responses. Onco Targets Ther. 2019;12:10903-10916.

27. Li T, Fan J, Wang B, et al. TIMER: a web server for comprehensive analysis of tumor-infiltrating immune cells. Cancer Res. 2017;77(21): e108-e110.

28. Patel S, Leal AD, Gorski DH. The homeobox gene Gax inhibits angiogenesis through inhibition of nuclear factor-kappaB-dependent endothelial cell gene expression. Cancer Res. 2005;65(4):1414-1424.

29. Whanger PD. Selenium and its relationship to cancer: an update. $\mathrm{Br}$ J Nutr. 2004;91(1):11-28.

30. Drutel A, Archambeaud F, Caron P. Selenium and the thyroid gland: more good news for clinicians. Clin Endocrinol (Oxf). 2013;78 (2):155-164.

31. Chen Y-C, Prabhu KS, Mastro AM. Is selenium a potential treatment for cancer metastasis? Nutrients. 2013;5(4):1149-1168.

32. Burk RF, Hill KE, Motley AK. Selenoprotein metabolism and function: evidence for more than one function for selenoprotein P. J Nutr. 2003;133(5Suppl 1):1517S-1520S.

33. Olson GE, Winfrey VP, Nagdas SK, Hill KE, Burk RF. Apolipoprotein E receptor-2 (ApoER2) mediates selenium uptake from selenoprotein P by the mouse testis. J Biol Chem. 2007;282 (16):12290-12297.

34. Burk RF, Hill KE, Motley AK, et al. Selenoprotein P and apolipoprotein E receptor-2 interact at the blood-brain barrier and also within the brain to maintain an essential selenium pool that protects against neurodegeneration. FASEB J. 2014;28(8):3579-3588.

35. Olson GE, Winfrey VP, Hill KE, Burk RF. Megalin mediates selenoprotein $\mathrm{P}$ uptake by kidney proximal tubule epithelial cells. $J$ Biol Chem. 2008;283(11):6854-6860.

36. Calvo A, Xiao N, Kang J, et al. Alterations in gene expression profiles during prostate cancer progression: functional correlations to tumorigenicity and down-regulation of selenoprotein-P in mouse and human tumors. Cancer Res. 2002;62(18):5325-5335.

37. Al-Taie OH, Uceyler N, Eubner U, et al. Expression profiling and genetic alterations of the selenoproteins GI-GPx and SePP in colorectal carcinogenesis. Nutr Cancer. 2004;48:1.

38. Murawaki Y, Tsuchiya H, Kanbe T, et al. Aberrant expression of selenoproteins in the progression of colorectal cancer. Cancer Lett. 2008;259(2):218-230.

39. Hughes DJ, Duarte-Salles T, Hybsier S, et al. Prediagnostic selenium status and hepatobiliary cancer risk in the European prospective investigation into cancer and nutrition cohort. Am J Clin Nutr. 2016;104(2):406-414.

40. Meyer HA, Endermann T, Stephan C, et al. Selenoprotein P status correlates to cancer-specific mortality in renal cancer patients. PLoS One. 2012;7(10):e46644.

41. Short SP, Williams CS. Selenoproteins in tumorigenesis and cancer progression. Adv Cancer Res. 2017;136:49-83.

42. Sato N, Fukushima N, Chang R, Matsubayashi H, Goggins M. Differential and epigenetic gene expression profiling identifies frequent disruption of the RELN pathway in pancreatic cancers. Gastroenterology. 2006;130(2):548-565.

International Journal of General Medicine

\section{Publish your work in this journal}

The International Journal of General Medicine is an international, peer-reviewed open-access journal that focuses on general and internal medicine, pathogenesis, epidemiology, diagnosis, monitoring and treatment protocols. The journal is characterized by the rapid reporting of reviews, original research and clinical studies

Submit your manuscript here: https://www.dovepress.com/international-journal-of-general-medicine-journal
43. Zhang Y, Zhou Y, Schweizer U, et al. Comparative analysis of selenocysteine machinery and selenoproteome gene expression in mouse brain identifies neurons as key functional sites of selenium in mammals. $J$ Biol Chem. 2008;283(4):2427-2438.

44. Yue C, Shan Z, Tan Y, et al. His-rich domain of selenoprotein $\mathrm{P}$ ameliorates neuropathology and cognitive deficits by regulating TrkB pathway and zinc homeostasis in an Alzheimer model of mice. ACS Chem Neurosci. 2020;11(24):4098-4110.

45. Solovyev N, Drobyshev E, Bjørklund G, Dubrovskii Y, Lysiuk R, Rayman MP. Selenium, selenoprotein P, and Alzheimer's disease: is there a link? Free Radic Biol Med. 2018;127:124-133.

46. Hoffmann PR, Berry MJ. The influence of selenium on immune responses. Mol Nutr Food Res. 2008;52(11):1273-1280.

47. Barrett CW, Singh K, Motley AK, et al. Dietary selenium deficiency exacerbates DSS-induced epithelial injury and AOM/DSS-induced tumorigenesis. PLoS One. 2013;8(7):e67845.

48. Yi Y-S, Park SG, Byeon SM, Kwon Y-G, Jung G. Hepatitis B virus $\mathrm{X}$ protein induces TNF-alpha expression via down-regulation of selenoprotein $\mathrm{P}$ in human hepatoma cell line, HepG2. Biochim Biophys Acta. 2003;1638(3):249-256.

49. Cat B, Stuhlmann D, Steinbrenner H, et al. Enhancement of tumor invasion depends on transdifferentiation of skin fibroblasts mediated by reactive oxygen species. J Cell Sci. 2006;119(Pt 13):2727-2738.

50. Bosschaerts T, Guilliams M, Noel W, et al. Alternatively activated myeloid cells limit pathogenicity associated with African trypanosomiasis through the IL-10 inducible gene selenoprotein P. J Immunol. 2008;180(9):6168-6175.

51. Short SP, Pilat JM, Williams CS. Roles for selenium and selenoprotein $\mathrm{P}$ in the development, progression, and prevention of intestinal disease. Free Radic Biol Med. 2018;127:26-35.

52. Speckmann B, Bidmon H-J, Borchardt A, Sies H, Steinbrenner H. Intestinal selenoprotein $\mathrm{P}$ in epithelial cells and in plasma cells. Arch Biochem Biophys. 2014;541:30-36.

53. Richman EL, Chan JM. Selenium and prostate cancer: the puzzle isn't finished yet. Am J Clin Nutr. 2012;96(1):1-2.

54. Pohl NM, Tong C, Fang W, Bi X, Li T, Yang W. Transcriptional regulation and biological functions of selenium-binding protein 1 in colorectal cancer in vitro and in nude mouse xenografts. PLoS One. 2009;4(11):e7774.

55. Streicher KL, Sylte MJ, Johnson SE, Sordillo LM. Thioredoxin reductase regulates angiogenesis by increasing endothelial cell-derived vascular endothelial growth factor. Nutr Cancer. 2004;50(2):221-231.

56. Lu J, Jiang C. Antiangiogenic activity of selenium in cancer chemoprevention: metabolite-specific effects. Nutr Cancer. 2001;40 (1):64-73. across all disease areas. The manuscript management system is completely online and includes a very quick and fair peer-review system, which is all easy to use. Visit http://www.dovepress.com/ testimonials.php to read real quotes from published authors. 